\title{
Solutions for Nonlinear Partial Differential Equations By Tan-Cot Method
}

\author{
Mahmood Jawad Abdul Rasool Abu Al-Shaeer \\ Al-Rafidain University College, Baghdad, Iraq
}

\begin{abstract}
In this paper, we established a traveling wave solution by using the proposed Tan-Cot function algorithm for nonlinear partial differential equations. The method is used to obtain new solitary wave solutions for various type of nonlinear partial differential equations such as, the (1+1)-dimensional Ito equation, Pochhammer-Chree (PC) equation, MIKP equation, Konopelchenko and Dubrovsky (KD) system of equations which are the important Soliton equations. Proposed method has been successfully implemented to establish new solitary wave solutions for the nonlinear PDEs.
\end{abstract}

Keywords: Nonlinear PDEs, Exact Solutions, Tan-Cot function method.

\section{INTRODUCTION}

The exact solutions of nonlinear partial differential equations (NLPDEs) play an important role in the study of many physical phenomena. With the help of exact solutions, when they exist, the mechanism of complicated physical phenomena and dynamical processes modeled by these NLPDEs can be better understood. They can also help to analyze the stability of these solutions and to check numerical analysis for these NLPDEs. Large varieties of physical, chemical, and biological phenomena are governed by nonlinear partial differential equations. One of the most exciting advances of nonlinear science and theoretical physics has been the development of methods to look for exact solutions of nonlinear partial differential equations [1]. Exact solutions to nonlinear partial differential equations play an important role in nonlinear science, especially in nonlinear physical science since they can provide much physical information and more insight into the physical aspects of the problem and thus lead to further applications. Nonlinear wave phenomena of dispersion, dissipation, diffusion, reaction and convection are very important in nonlinear wave equations. In recent years, quite a few methods for obtaining explicit traveling and solitary wave solutions of nonlinear evolution equations have been proposed. A variety of powerful methods, such as, tanh-sech method [2], extended tanh method [3], hyperbolic function method [4], Jacobi elliptic function expansion method [5], F-expansion method [6], and the First Integral method [7]. The sine-cosine method [8] has been used to solve different types of nonlinear systems of PDEs.

In this paper, we applied the Tan-Cot method to solve the (1+1)-dimensional Ito equation, PochhammerChree (PC) equation, MIKP equation, Konopelchenko and Dubrovsky KD) system of equations, given respectively by:

$\mathrm{u}_{\mathrm{tt}}+\mathrm{u}_{\mathrm{xxxt}}+3\left(2 \mathrm{u}_{\mathrm{x}} \mathrm{u}_{\mathrm{t}}+\mathrm{uu}_{\mathrm{xt}}\right)+3 \mathrm{u}_{\mathrm{xx}} \int_{-\infty}^{\mathrm{x}} \mathrm{u}_{\mathrm{t}} \mathrm{dx}=0$
$\mathrm{u}_{\mathrm{tt}}-\mathrm{u}_{\mathrm{ttxx}}-\left(\mathrm{a} \mathrm{u}-\mathrm{b} \mathrm{u}^{3}\right)_{\mathrm{xx}}=0$
$u_{t x}+2 u u_{x}^{2}+u^{2} u_{x x}+a u_{x x x}+b u_{y y}=0$
$u_{t}-6 b u u_{x}-u_{x x x}+\frac{3}{2} a^{2} u^{2} u_{x}-3 v_{y}+3 a u_{x} v=0 \quad, u_{y}=v_{x}$

\section{The TAN-COT FunCTION METHOD}

Consider the nonlinear partial differential equation in the form [9]

$F\left(u, u_{t}, u_{x}, u_{y}, u_{t t}, u_{x x}, u_{x y}, u_{y y}, \ldots \ldots \ldots \ldots\right)=0$

where $\mathrm{u}(\mathrm{x}, \mathrm{y}, \mathrm{t})$ is a traveling wave solution of nonlinear partial differential equation Eq. (5). We use the transformations,

$u(x, y, t)=f(\xi)$

where $\xi=k x+\delta y-\lambda t$ This enables us to use the following changes:

$\frac{\partial}{\partial t}()=.-\lambda \frac{d}{d \xi}(),. \frac{\partial}{\partial x}()=.k \frac{d}{d \xi}(),. \frac{\partial}{\partial y}()=.\delta \frac{d}{d \xi}($.

Using Eq. (7) to transfer the nonlinear partial differential equation Eq. (5) to nonlinear ordinary differential equation

$Q\left(f, f^{\prime}, f^{\prime \prime}, f^{\prime \prime \prime}, \ldots \ldots \ldots \ldots . . . ..\right)=0$

The ordinary differential equation (8) is then integrated as long as all terms contain derivatives, where we neglect the integration constants. The solutions of many nonlinear equations can be expressed in the form [9]: 
$f(\xi)=\alpha \tan ^{\beta}(\mu \xi), \quad|\xi| \leq \frac{\pi}{2 \mu}$

or in the form

$f(\xi)=\alpha \cot ^{\beta}(\mu \xi), \quad|\xi| \leq \frac{\pi}{2 \mu}$

Where $\alpha, \mu$, and $\beta$ are parameters to be determined, We use

$f(\xi)=\alpha \tan ^{\beta}(\mu \xi)$

$f^{\prime}=\alpha \beta \mu\left[\tan ^{\beta-1}(\mu \xi)+\tan ^{\beta+1}(\mu \xi)\right]$

$f^{\prime \prime}=\alpha \beta \mu^{2}\left[(\beta-1) \tan \beta-2(\mu \xi)+2 \beta \tan ^{\beta}(\mu \xi)+(\beta+1) \tan \beta+2(\mu \xi)\right]$

$f^{\prime \prime \prime}=\beta \mu^{3} \alpha\left[(\beta-1)(\beta-2) \tan ^{\beta-3}(\mu \xi)+\left(3 \beta^{2}-3 \beta+2\right) \tan ^{\beta-1}(\mu \xi)+(\beta+1)(\beta+2) \tan ^{\beta}(\mu \xi)+\right.$

$\left.2 \beta^{2} \tan ^{\beta+1}(\mu \xi)+(\beta+1)(\beta+2) \tan ^{\beta}+2(\mu \xi)\right]$

and their derivative. Or use

$\mathrm{f}(\xi)=\alpha \cot ^{\beta}(\mu \xi)$

$f^{\prime}=-\alpha \beta \mu\left[\cot ^{\beta-1}(\mu \xi)+\cot ^{\beta+1}(\mu \xi)\right]$

$f^{\prime \prime}=\alpha \beta \mu^{2}\left[(\beta-1) \cot ^{\beta-2}(\mu \xi)+2 \beta \cot ^{\beta}(\mu \xi)+(\beta+1) \cot ^{\beta+2}(\mu \xi)\right]$

and so on. We substitute Eq.(10) or Eq.(11) into the reduced equation (8), balance the terms of the tan functions when Eq. (10) are used, or balance the terms of the cot functions when Eq. (11) are used, and solve the resulting system of algebraic equations by using computerized symbolic packages. We next collect all terms with the same power in $\tan ^{k}(\mu \xi)$ or $\cot ^{k}(\mu \xi)$ and set to zero their coefficients to get a system of algebraic equations among the unknown's $\alpha, \mu$ and $\beta$, and solve the subsequent system.

\section{Applications}

In this section we apply the Tan-Cot method to different nonlinear partial differential equations:

\section{The (1+1)-dimensional Ito equation}

Consider The (1+1)-dimensional Ito equation [10]:

$\mathrm{u}_{\mathrm{tt}}+\mathrm{u}_{\mathrm{xxxt}}+3\left(2 \mathrm{u}_{\mathrm{x}} \mathrm{u}_{\mathrm{t}}+\mathrm{uu}_{\mathrm{xt}}\right)+3 \mathrm{u}_{\mathrm{xx}} \int_{-\infty}^{\mathrm{x}} \mathrm{u}_{\mathrm{t}} \mathrm{dx}=0$

Assume:

$\mathrm{u}(\mathrm{x}, \mathrm{t})=\mathrm{v}_{\mathrm{x}}(\mathrm{x}, \mathrm{t})$

Then Eq.(12) can be written as:

$\mathrm{v}_{\mathrm{ttx}}+\mathrm{v}_{\mathrm{xxxxt}}+3\left(2 \mathrm{v}_{\mathrm{xx}} \mathrm{v}_{\mathrm{xt}}+\mathrm{v}_{\mathrm{x}} \mathrm{v}_{\mathrm{xxt}}\right)+3 \mathrm{v}_{\mathrm{xxx}} \mathrm{v}_{\mathrm{t}}=0$

we introduce the transformations

$\xi=\mathrm{kx}-\lambda \mathrm{t}$

where $\mathrm{k}$, and $\lambda$ are real constants. Equation (14) becomes

$\lambda \mathrm{v}^{\prime \prime \prime}-\mathrm{k}^{3} \mathrm{v}^{(5)}-3 \mathrm{k}^{2}\left[\mathrm{v}^{\prime 2}\right]^{\prime \prime}=0$

Integrating Eq. (16) twice with zero constants, we get

$\lambda \mathrm{v}^{\prime}-\mathrm{k}^{3} \mathrm{v}^{\prime \prime \prime}-3 \mathrm{k}^{2}\left(\mathrm{v}^{\prime}\right)^{2}=0$

$\mathrm{w}(\xi)=\mathrm{v}^{\prime}(\xi)$

Eq.(18) becomes

$\lambda \mathrm{w}-\mathrm{k}^{3} \mathrm{w}^{\prime \prime}-3 \mathrm{k}^{2} \mathrm{w}^{2}=0$

Applying the tan function method as in Eq.(10), then Eq.(19) becomes

$\lambda \alpha \tan ^{\beta}(\mu \xi)-\mathrm{k}^{3} \alpha \beta \mu^{2}\left[(\beta-1) \tan ^{\beta-2}(\mu \xi)+2 \beta \tan ^{\beta}(\mu \xi)+(\beta+1) \tan ^{\beta+2}(\mu \xi)\right]-$

$3 \mathrm{k}^{2} \alpha^{2} \tan ^{2 \beta}(\mu \xi)=0$

Then Eq.(20) can written as

$\lambda \tan ^{\beta}(\mu \xi)-\mathrm{k}^{3} \beta \mu^{2}\left[(\beta-1) \tan ^{\beta-2}(\mu \xi)+2 \beta \tan ^{\beta}(\mu \xi)+(\beta+1) \tan ^{\beta+2}(\mu \xi)\right]-3 \mathrm{k}^{2} \alpha \tan ^{2 \beta}(\mu \xi)=0$

Balancing the exponents $\beta+2$ and $2 \beta$ then

$\beta+2=2 \beta$ and we get $\beta=2$

Substitute $\beta=2$ in Eq. (21) to get the value of :

$\lambda=8 \mathrm{k}^{3} \mu^{2}, \alpha=-2 \mathrm{k} \mu^{2}$

Then :

$\mathrm{w}(\xi)=-2 \mathrm{k} \mu^{2} \tan ^{2}(\mu \xi)$

Integrate Eq.(22) for $\xi$ to get:

$\mathrm{v}(\mathrm{x}, \mathrm{t})=-2 \mathrm{k} \mu[\tan (\mu(\mathrm{kx}-\lambda \mathrm{t}))-\mu(\mathrm{kx}-\lambda \mathrm{t})]$

From Eq. (23) we get:

$\mathrm{u}(\mathrm{x}, \mathrm{t})=\mathrm{v}_{\mathrm{x}}=-2 \mathrm{k}^{2} \mu^{2} \tan ^{2}\left(\mu \mathrm{k}\left(\mathrm{x}-8 \mathrm{k}^{2} \mu^{2} \mathrm{t}\right)\right)$ 
For $\mu=\mathrm{k}=1$, Eq.(24) becomes:

$\mathrm{u}(\mathrm{x}, \mathrm{t})=-2 \tan ^{2}(\mathrm{x}-8 \mathrm{t})$

$u(x, t)$ in (25) is represented in Figure (1) for $-10 \leq x \leq 10$ and $0 \leq t \leq 1$.

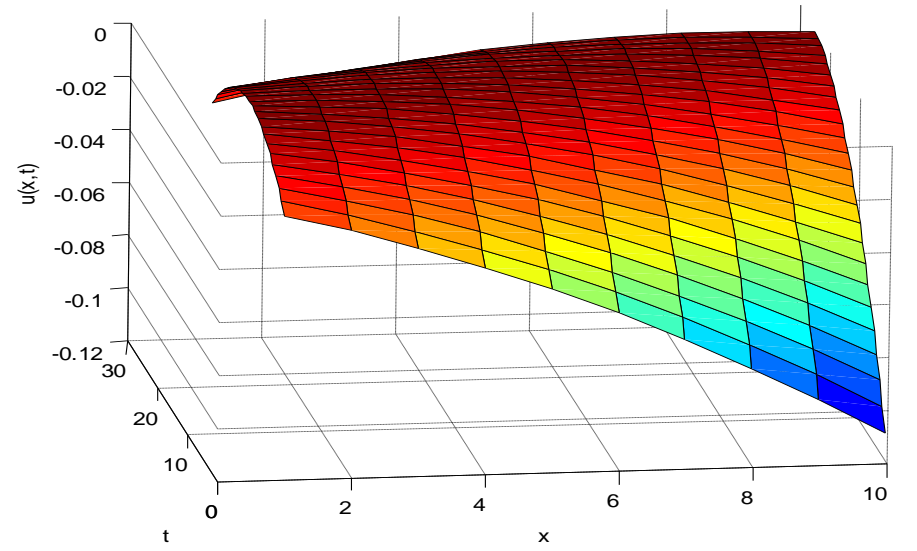

Figure (1) represent $u(x, t)$ in (25) for $-10 \leq x \leq 10$ and $0 \leq t \leq 1$.

\section{Pochhammer-Chree (PC) equation}

Consider the Pochhammer-Chree (PC) equation [11]

$u_{t t}-u_{t t x x}-\left(a u-b u^{3}\right)_{x x}=0$

We introduce the transformation $\xi=(k x-\lambda t)$, where $\mathrm{k}$, and $\lambda$ are real constants. Equation (26) transforms to the ODE:

$\lambda^{2} u^{\prime \prime}-\lambda^{2} k^{2} u^{(4)}-k^{2}\left(a u-b u^{3}\right) "=0$

Integrating Eq.(27) twice with zero constant to get the following ordinary differential equation:

$\lambda^{2} u-\lambda^{2} k^{2} u^{\prime \prime}-k^{2}\left(a u-b u^{3}\right)=0$

Seeking the solution in Eq.(11)

$\lambda^{2} \alpha \cot ^{\beta}(\mu \xi)-\lambda^{2} k^{2} \alpha \beta \mu^{2}\left[(\beta-1) \cot ^{\beta-2}(\mu \xi)+2 \beta \cot ^{\beta}(\mu \xi)+(\beta+1) \cot ^{\beta+2}(\mu \xi)\right]-$

$k^{2} a \alpha \cot ^{\beta}(\mu \xi)+k^{2} b \alpha^{3} \cot ^{3 \beta}(\mu \xi)=0$

Equating the exponents and the coefficients of each pair of the cot functions we find the following algebraic system:

$3 \beta=\beta+2 \rightarrow \beta=1$

Substituting Eq. (30) into Eq. (29) to get:

$\lambda^{2} \cot (\mu \xi)-2 \lambda^{2} k^{2} \mu^{2}\left[\cot (\mu \xi)+\cot ^{3}(\mu \xi)\right]-k^{2} a \cot (\mu \xi)+k^{2} b \alpha^{2} \cot ^{3}(\mu \xi)=0$

Equating the exponents and the coefficients of each pair of the cot function, we obtain a system of algebraic equations:

$\cot (\mu \xi): \lambda^{2}-2 \lambda^{2} k^{2} \mu^{2}-k^{2} a=0$

$\cot ^{3}(\mu \xi):-2 \lambda^{2} k^{2} \mu^{2}+k^{2} b \alpha^{2}=0$

By solving the algebraic system (32), we get,

$\lambda=k \sqrt{\frac{a}{\left[1-2 k^{2} \mu^{2}\right]}}, \alpha=\sqrt{\frac{2 a}{b\left[1-2 k^{2} \mu^{2}\right]}} \mathrm{k} \mu$

Then by substituting Eq.(33) into Eq.(11), the exact soliton solution of equation (26) can be written in the form

$u(x, t)=\sqrt{\frac{2 a}{b\left[1-2 k^{2} \mu^{2}\right]}} \mathrm{k} \mu \cot \left(\mu k\left(x-\sqrt{\frac{a}{\left[1-2 k^{2} \mu^{2}\right]}} t\right)\right), \quad 0<\mu k\left(x-\sqrt{\frac{a}{\left[1-2 k^{2} \mu^{2}\right]}} t\right)<\pi$

For , $\mu=k=\varepsilon=1$, then (34) becomes:

$u(x, t)=\sqrt{\frac{-2 a}{b}} \cot ((x-\sqrt{-a} t)), a<0, b>0$

For , $a=-1, b=1$, then (35) becomes:

$u(x, t)=\sqrt{2} \cot (x-t)$

$u(x, t)$ in (36) is represented in Figure (2) for $-10 \leq x \leq 10$ and $0 \leq t \leq 1$. 


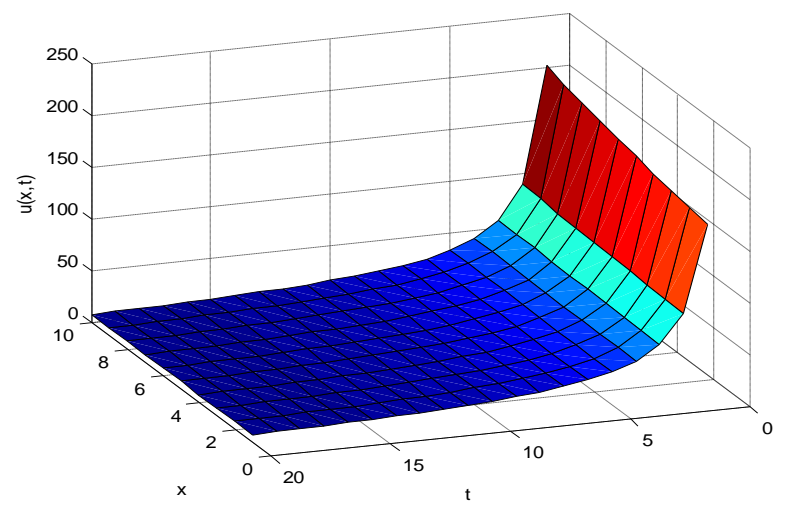

Figure (2). Represents $u(x, t)$ in (36) for $0 \leq x \leq 10$ and $0 \leq t \leq 1$.

\section{MIKP equation}

\section{Consider the MIKP equation [12]}

$u_{t x}+2 u u_{x}^{2}+u^{2} u_{x x}+a u_{x x x}+b u_{y y}=0$

Let us now solve Eq.(37) by the proposed method. We introduce the transformation

$u(x, y, t)=U(\xi), \quad \xi=k x+l y-\lambda t$

, where $\mathrm{k}$, and $\lambda$ are real constants. Equation (38) transforms to the ODE:

$-k \lambda u^{\prime \prime}+k^{2}\left[2 u u^{2}+u^{2} u^{\prime \prime}\right]+a k^{4} u^{\prime \prime \prime \prime}+b l^{2} u^{\prime \prime}=0$

Eq.(39) can be written as

$-k \lambda u^{\prime \prime}+\frac{k^{2}}{3}\left[u^{3}\right]^{\prime \prime}+a k^{4} u^{\prime \prime \prime \prime}+b l^{2} u^{\prime \prime}=0$

Integrating (40) once with zero constant to get the following ordinary differential equation:

$\left(b l^{2}-k \lambda\right) u+\frac{k^{2}}{3} u^{3}+a k^{4} u^{\prime \prime}=0$

Seeking the solution in (11)

$\left(b l^{2}-k \lambda\right) \alpha \cot ^{\beta}(\mu \xi)+\frac{k^{2}}{3} \alpha^{3} \cot ^{3 \beta}(\mu \xi)+a k^{4} \alpha \beta \mu^{2}\left[(\beta-1) \cot ^{\beta-2}(\mu \xi)+2 \beta \cot ^{\beta}(\mu \xi)+\right.$ $\left.(\beta+1) \cot ^{\beta+2}(\mu \xi)\right]=0$

(42)

Equating the exponents and the coefficients of each pair of the cot functions we find the following algebraic system:

$3 \beta=\beta+2 \rightarrow \beta=1$

$\left(b l^{2}-k \lambda\right) \cot (\mu \xi)+\frac{k^{2}}{3} \alpha^{2} \cot ^{3}(\mu \xi)+2 a k^{4} \mu^{2}\left[\cot (\mu \xi)+\cot ^{3}(\mu \xi)\right]=0$

$\cot ^{3}(\mu \xi) \quad: \frac{k^{2}}{3} \alpha^{2}+2 a k^{4} \quad \mu^{2}=0$

$\cot ^{1}(\mu \xi):\left(b l^{2}-k \lambda\right)+2 a k^{4} \mu^{2}=0$

By solving the algebraic system (44), we get,

$\alpha=\frac{\mathrm{i}}{\mathrm{k}} \sqrt{3\left(\lambda k-b l^{2}\right)}, \mu=\sqrt{\frac{\lambda k-b l^{2}}{2 a k^{4}}}$

Then by substituting Eq. (45) into Eq. (11), the exact soliton solution of equation (37) can be written in the form

$u(x, t)=\frac{\mathrm{i}}{\mathrm{k}} \sqrt{3\left(\lambda k-b l^{2}\right)} \cot \left\{\sqrt{\frac{\lambda k-b l^{2}}{2 a k^{4}}}(k x+l y-\lambda t)\right\}, 0<\sqrt{\frac{\lambda k-b l^{2}}{2 a k^{4}}}(k x+l y-\lambda t)<\pi$

For $\lambda=k=l=1, a=b=1 / 2$ Eq.(46) becomes

$u(x, t)=\mathrm{i} \sqrt{\frac{3}{2}} \cot \left\{\sqrt{\frac{1}{2}}(x+y-t)\right\}$

$u(x, t)$ in (47) is represented in figure (3) for $0 \leq x \leq 10$ and $y=1$, and $0 \leq t \leq 1$. 


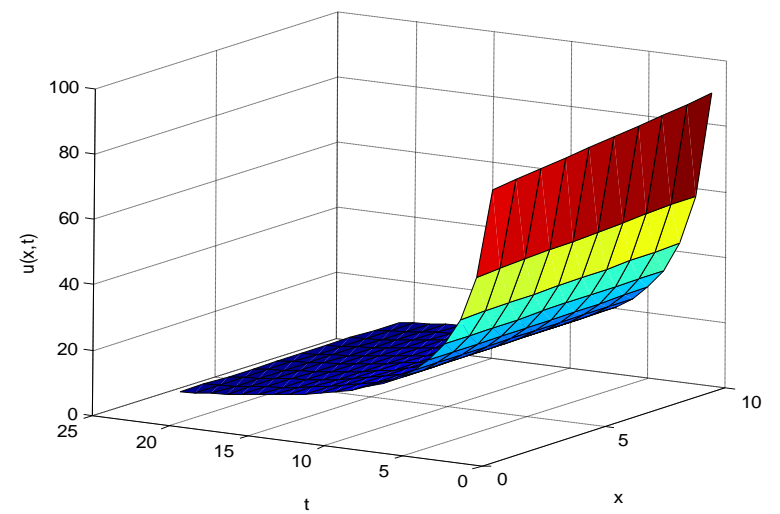

Figure (3). Represents $u(x, t)$ in (46) for $0 \leq x \leq 10$ and $0 \leq t \leq 1$.

\section{Konopelchenko-Dubrovsky (KD) equation}

Konopelchenko and Dubrovsky (1984) presented the Konopelchenko-Dubrovsky (KD) equation[13] $u_{t}-6 b u u_{x}-u_{x x x}+\frac{3}{2} a^{2} u^{2} u_{x}-3 v_{y}+3 a u_{x} v=0$ $u_{y}=v_{x}$

where $a$ and $b$ are real parameters. Equations (48), and (49) is a new nonlinear integrable evolution equation on two spatial dimensions and one temporal. This equation was investigated by the inverse scattering transform method. The F-expansion method is also used in Wang and Zhang [14] to investigate the KD equation.

To solve Eqs.(48), and (49) by the proposed method. We introduce the transformation $\xi=(k x+l y-\lambda t)$, where $k, l$, and $\lambda$ are real constants. Equation (48) transforms to the ODE:

$-\lambda u^{\prime}-6 k b u u^{\prime}-k^{3} u^{\prime \prime \prime}+\frac{3}{2} k a^{2} u^{2} u^{\prime}-3 l v^{\prime}+3 k a u^{\prime} v=0$

And Eq.(49) transforms to:

$v^{\prime}=\frac{l u^{\prime}}{k}$

Integrating Eq.(51) with zero constant

$v=\frac{l}{k} u$

Substitute Eqs.(51) and (52) in Eq.(50) to get

$\left[-\lambda k-3 l^{2}\right] u^{\prime}-k^{3} u^{\prime \prime \prime}-\frac{3}{2} k[2 k b-l a]\left[u^{2}\right]^{\prime}+\frac{1}{2} k^{2} a^{2}\left[u^{3}\right]^{\prime}=0$

Integrating $\mathrm{Eq}(53)$ once with zero constant to get

$-\left[\lambda k+3 l^{2}\right] u-k^{3} u^{\prime \prime}-\frac{3}{2} k[2 k b-l a] u^{2}+\frac{1}{2} k^{2} a^{2} u^{3}=0$

Seeking the solution in (10)

$-\left[\lambda k+3 l^{2}\right] \alpha \tan ^{\beta}(\mu \xi)-k^{3} \alpha \beta \mu^{2}\left[(\beta-1) \tan ^{\beta-2}(\mu \xi)+2 \beta \tan ^{\beta}(\mu \xi)+(\beta+\right.$

1) $\left.\tan ^{\beta+2}(\mu \xi)\right]-\frac{3}{2} k[2 k b-l a] \alpha^{2} \tan ^{2 \beta}(\mu \xi)+\frac{1}{2} k^{2} a^{2} \alpha^{3} \tan ^{3} \beta(\mu \xi)=0$

From (55), equating exponents $\beta+2$ and $3 \beta$ yield

$\beta+2=3 \beta$, so that $\beta=1$

then Eq.(56) becomes:

$-\left[\lambda k+3 l^{2}\right] \tan (\mu \xi)-2 k^{3} \mu^{2}\left[\tan (\mu \xi)+\tan ^{3}(\mu \xi)\right]-\frac{3}{2} k[2 k b-l a] \alpha \tan ^{2}(\mu \xi)+$

$\frac{1}{2} k^{2} a^{2} \alpha^{2} \tan ^{3}(\mu \xi)=0$

Balancing the same exponents to give:

$\tan (\mu \xi):-\left[\lambda k+3 l^{2}\right]-2 k^{3} \mu^{2}=0$

$\tan ^{2}(\mu \xi):-\frac{3}{2} k[2 k b-l a] \alpha=0$

$\tan ^{3}(\mu \xi):-2 k^{3} \mu^{2}+\frac{1}{2} k^{2} a^{2} \alpha^{2}=0$

By solving the algebraic system (58), we get,

$\lambda=-2 k \frac{6 b^{2}+k a^{2} \mu^{2}}{a^{2}}, l=\frac{2 k b}{a} \quad, \alpha=\mp \frac{2 \mu}{a} \sqrt{k}$

Then by substituting Eq. (59) into Eq. (10), the exact soliton solution of equation (54) can be written in the form

$u(x, t)=\mp 2 \frac{\mu}{a} \sqrt{k} \tan \left[\mu k\left(x+\frac{2 b}{a} y+2 \frac{6 b^{2}+k a^{2} \mu^{2}}{a^{2}} t\right)\right]$ 
$v(x, t)=\mp 4 \frac{\sqrt{k} b \mu}{a^{2}} \tan \left[\mu k\left(x+\frac{2 b}{a} y+2 \frac{6 b^{2}+k a^{2} \mu^{2}}{a^{2}} t\right)\right]$

for $\mu=k=a=b=1$

$u(x, t)=\mp 2 \tan [(x+2 y+14 t)]$

$v(x, t)=\mp 4 \tan [(x+2 y+14 t)]$

Figures (4) represents $u(x, t)$ in (62), for $0 \leq x \leq 10, y=1$, and $0.1 \leq t \leq 1$.

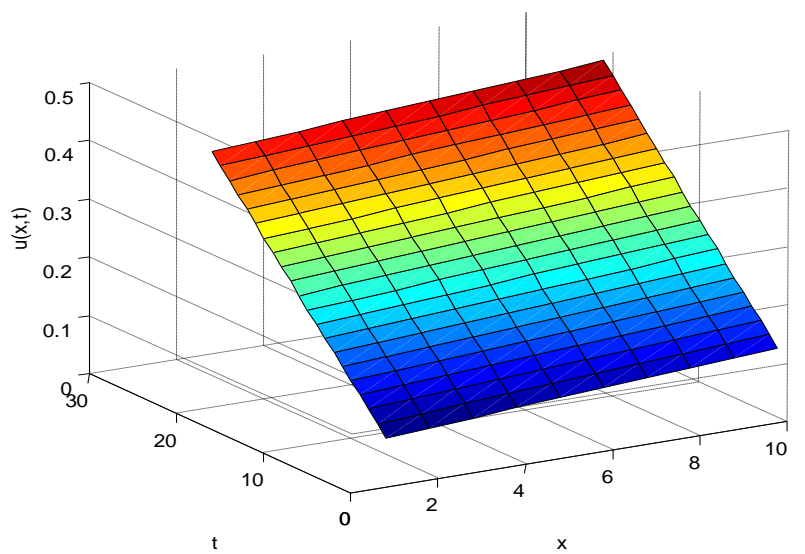

Figure (4) represents $u(x, t)$ in (61) for $0 \leq x \leq 10$ and $0.1 \leq t \leq 1$.

\section{Conclusion}

In this paper, the Tan-Cot function method has been implemented to establish new solitary wave solutions for various types of nonlinear PDEs. We can say that the new method can be extended to solve the problems of nonlinear partial differential equations which arising in the theory of solitons and other areas.

\section{References}

[1] Marwan Alquran, Kamel Al-Khaled, Hasan Ananbeh (2011). New Soliton Solutions for Systems of Nonlinear Evolution Equations by the Rational Sine-Cosine Method. Studies in Mathematical Sciences, Vol. 3, No. 1, pp. 1-9.

[2] Malfliet, W. (1992). Solitary wave solutions of nonlinear wave equations. Am. J. Phys, Vol. 60, No. 7, pp. 650-654

[3] El-Wakil, S.A, Abdou, M.A. (2007). New exact travelling wave solutions using modified extended tanh-function method, Chaos Solitons Fractals, Vol. 31, No. 4, pp. 840-852.

[4] Xia, T.C., Li, B. and Zhang, H.Q. (2001). New explicit and exact solutions for the Nizhnik- Novikov-Vesselov equation, Appl. Math. E-Notes, Vol. 1, pp. 139-142.

[5] Inc, M., Ergut, M. (2005). Periodic wave solutions for the generalized shallow water wave equation by the improved Jacobi elliptic function method, Appl. Math. E-Notes, Vol. 5, pp. 89-96.

[6] Zhang, Sheng. (2006). The periodic wave solutions for the (2+1) -dimensional Konopelchenko Dubrovsky equations, Chaos Solitons Fractals, Vol. 30, pp. 1213-1220.

[7] Feng, Z.S. (2002). The first integer method to study the Burgers-Korteweg-de Vries equation, J Phys. A. Math. Gen, Vol. 35, No. 2, pp. 343-349.

[8] Mitchell A. R. and D. F. Griffiths (1980), The Finite Difference Method in Partial Differential Equations, John Wiley \& Sons.

[9] Anwar J. M. Jawad, (2012), New Exact Solutions of Nonlinear Partial Differential Equations Using Tan-Cot Function Method, Studies in Mathematical Sciences Vol. 5, No. 2, pp. 13-25.

[10] A.M. Wazwaz, Multiple-soliton solutions for the generalized (1+1)-dimensional and the generalized (2+1)-dimensional Ito equations, Appl. Math. Comput. 202 (2008): 840-849.

[11] Biao Li, Yong Chen, and Hongqing Zhang, Travelling Wave Solutions for Generalized Pochhammer-Chree Equations, Verlag der Zeitschrift für Naturforschung, Tu“bingen " www.znaturforsch.com

[12] N. Taghizadeh and S.R. Moosavi Noori, Two Reliable Methods for Solving the Modified Improved Kadomtsev-Petviashvili Equation, Applications and Applied Mathematics Vol. 7, Issue 2 (December 2012), pp. 658 - 671.

[13] N. Taghizadeh and M. Mirzazadeh, Modification of Truncated Expansion Method for Solving Some Important Nonlinear Partial Differential Equations, Applications and Applied Mathematics Vol. 7, Issue 2 (December 2012), pp. 488 - 499.

[14] Wang, D., Zhang, H.-Q. (2005). Further improved F-expansion method and new exact solutions of Konopelchenko-Dubrovsky equation, Chaos, Solitons and Fractals, Vol. 25, pp. 601-610. 